\title{
Evaluation of Resistance against Vibration and Shock Resistance on Spherical Ultrasonic Motor
}

\author{
Uichi Nishizawa, Taro Oohashi, and Shigeki Toyama
}

\begin{abstract}
The spherical ultrasonic motor which drives in the space environment has been developed by this research. It has been evaluated with regard to high temperature and low temperature which assumed the space environment as the previous studies. On the other hand, a vibration and a shock assumed at launch of a rocket are severe condition, but the spherical ultrasonic motor has to stand up to the vibration and the shock. So it was evaluated with regard to resistance against vibration and shock resistance to the spherical ultrasonic motor by this research. The natural frequency of the spherical ultrasonic motor was calculated and theoretical calculation about the breaking strength by the vibration was performed as evaluation method. And a result of the theoretical calculation was verified by a resonance search experiment and a stimules experiment. As a result, the resistance against vibration of the spherical ultrasonic motor was clarified. And the shock resistance of the spherical ultrasonic motor was confirmed to shock acceleration of $\mathbf{4 0} \mathbf{~ G}$.
\end{abstract}

Index Terms - Ultrasonic motor, resistance against vibration, shock resistance, natural frequency, space environment.

\section{INTRODUCTION}

In recent years, a space development business is tackled in earnest, and a lift-off of an artificial satellite is carried out actively. An artificial satellite veers off the orbit by influence of the solar wind and the perturbing force in an operating orbit. An orbital adjustment of an artificial satellite is performed by a jet of a thruster. An artificial satellite has a lot of thrusters, and the respective thrusters are turned to the various ways. When the direction of the thruster can be controlled, several thrusters can be integrated. And space-saving and weight saving of an artificial satellite can be expected. The spherical ultrasonic motor (SUSM) of the previous studies [1]-[4] is applied to the direction control of a thruster of an artificial satellite by this research. The SUSM is suitable as a motor for direction control, because there is 3 degree of freedom with regard to a revolution by itself.

It is evaluated about the high temperature environment and the low temperature environment which assumed a space environment to the SUSM by our previous studies [5]-[7]. On the other hand, a vibration and a shock assumed at launch of a rocket are severe condition, but the SUSM has to stand up to the vibration and the shock. So the purpose of this research is to evaluate resistance against vibration and shock resistance of SUSM. The natural frequency of the SUSM is calculated and theoretical calculation with respect to the breaking strength of the SUSM by the vibration is carried out

Manuscript received February 20, 2018; revised April 12, 2018.

Uichi Nishizawa and Shigeki Toyama are with Tokyo University of Agriculture and Technology, Koganei, Tokyo, Japan (e-mail: n-uichi@cc.tuat.ac.jp, toyama@cc.tuat.ac.jp). as evaluation method. And results of the theoretical calculation are verified by a resonance search experiment and a stimules experiment.

\section{CHARACTERISTICS OF SUSM}

The mechanical vibration of the ultrasonic range beyond $20 \mathrm{kHz}$ is used for drive of the ultrasonic motor [8], [9], and the piezoelectric element is glued by the metallic elastic body of the stator together. The piezoelectric element is divided into 11 parts, and consists of the phase $\mathrm{A}$, the phase $\mathrm{B}$, and the feedback phase. And the piezoelectric element expands and contracts in the direction of circumference by the impressed voltage, and bends in the direction of thickness. When sine wave alternating voltage of two kinds are impressed on phase A and phase B of the piezoelectric element, the traveling wave is generated into the piezoelectric element. The traveling wave on the circle surface of the metallic elastic body drives the rotor forced on the circle. When the phase difference of the input sine wave of phase A and phase B is changed, the rate of the traveling wave is changed. Therefore it is possible to control the rotational speed of the rotor by the phase difference of the inlet voltage.

The SUSM is shown in Fig. 1. Three stators are installed around the spherical rotor of the SUSM. When three rotation vector of the driving force generated by a stator is composed, a spherical rotor can be driven about any rotation axis. The direction of rotation is controlled by setting the phase difference of input sine wave alternating voltage of two kinds to each stator. The SUSM has the following characteristics as compared with DC motor: (a) having three degrees of freedom by oneself, (b) miniature of a motor is easy because the structure is simple, (c) having high torque in low speed range for direct drive by friction, (d) having high response, (e) the brake is not needed to have holding torque, (f) the electric field and the magnetic field do not influence because it is not drive by the electromagnetic force.

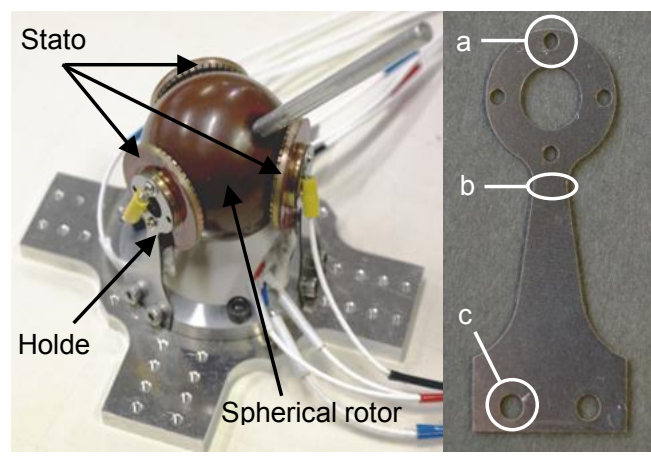

Fig. 1. SUSM (left) and holder (right). 


\section{SUSM FOR SPACE}

\section{A. Requirement Specification to Motor for Space}

The spherical rotor of the diameter $45 \mathrm{~mm}$ and the stators of diameter $30 \mathrm{~mm}$ are used by this research. The requirement specifications to the motor for space are as follows.

1) Small size and light weight.

2) Maintenance-free machines.

3) Driving in vacuum environment.

4) Operating temperature range is wide.

5) There is no discharge of outgassing.

6) There is no degradation by radiation.

7) Resistance against vibration and shock resistance are high.

The SUSM satisfies the specification of the 1) because the structure of the SUSM is easy. When 1 of 3 stators of the SUSM broke down, the SUSM can drive 3 degree of freedom by 2 stators. Therefore the SUSM satisfies the specification of the 2). The drive in vacuum environment of the specification 3) and the drive in high temperature environment and low temperature environment of the specification 4) are confirmed by the previous study. The SUSM satisfies the specification of the 5) because the material without the outgassing was selected in the component of the SUSM by the previous study.

\section{B. Resistance Against Vibration and Shock Resistance}

The SUSM satisfies the requirement specifications from above-mentioned 1) to 5), but it is also necessary to endure the vibration and the shock at launch of a rocket. An artificial satellite receives the quasi-static acceleration, the sine wave vibration, and the random vibration indicated in Table I-III for approximately 60 seconds as the vibrational load at launch of a rocket. Further, the vibrational load of Table I-III referred to the design load of the artificial satellite loaded into the Japanese H-IIA rocket.

TABLE I: QUASI-STATIC ACCELERATION OF H-IIA ROCKET

\begin{tabular}{l|l}
\hline \hline Axis direction & $6.0 \mathrm{G}$ \\
\hline Crossing at right angles direction & $5.0 \mathrm{G}$ \\
\hline \hline
\end{tabular}

TABLE II: SINE WAVE VIBRATION OF H-IIA ROCKET

\begin{tabular}{l|l|l}
\hline \hline & Frequency & Acceleration \\
\hline Axis direction & $5-100 \mathrm{~Hz}$ & $2.5 \mathrm{G}$ \\
\hline Crossing at right angles direction & $5-100 \mathrm{~Hz}$ & $2.0 \mathrm{G}$ \\
\hline \hline
\end{tabular}

TABLE III: RANDOM VIBRATION OF H-IIA ROCKET

\begin{tabular}{l|l|l}
\hline \hline Frequency $(\mathrm{Hz})$ & PSD $\left(\mathrm{G}^{2} / \mathrm{Hz}\right)$ & RMS $(\mathrm{G})$ \\
\hline $20-200$ & $+3 \mathrm{~dB} /$ oct & 7.8 \\
$200-2000$ & 0.032 & \\
\hline \hline
\end{tabular}

The quasi-static acceleration is the vibration by which the load caused by low frequency oscillation of a fuselage was added to the steady acceleration after launch. The sine wave vibration is vibration caused by vibration of a fuselage by various disturbances after launch. The random vibration is the vibration as which a pressure fluctuation acts on an artificial satellite through a fairing. In particular, when the sine wave vibration and the natural frequency of the SUSM resonate, amplitude by vibration of SUSM will be extremely big, and there is danger to which interference to other equipment occurs. The load will be big by the resonance magnification, and the SUSM causes the danger which breaks down. Therefore the natural frequency beyond $100 \mathrm{~Hz}$ is needed by the SUSM.

On the other hand, an artificial satellite receives the shock in separation from a rocket of an artificial satellite, opening and shutting of a fairing part, and development of a structure. The shock load which forms in the case is bigger than a vibrational load. In particular, the extremely large shock load is added to an artificial satellite for separation from a rocket of an artificial satellite and an explosion of the gunpowder when opening a fairing part. The SUSM should have the strength which does not just break down by these loads.

The natural frequency of the SUSM and resistance to the vibrational load indicated in Table I-III are evaluated theoretically and experimentally about resistance against vibration. On the other hand, it is evaluated by a stimules experiment about shock resistance.

\section{NATURAL FREQUENCY OF SUSM}

\section{A. Calculation of Natural Frequency of SUSM}

For the vibration assumed at a launch of a rocket to be added to the axle direction of a rocket and the crossing at right angles direction, the natural frequency of the SUSM is calculated to vibration in these 2 directions. So a calculation model was created to the SUSM indicated on Fig. 1 and a natural vibration analysis by the finite element method was performed. The underside on the screw hole for fixing of the base is the perfect fixture about the restrains to the calculation model indicated on Fig. 2, and it is not established because it is the natural vibration analysis about the load. In case of the SUSM, a spherical rotor is maintained by the frictional force which operates on a rubbing surface between the stator and the spherical rotor and the pressing power by the stiffness in the holder part. Therefore the combination condition of the stator and the spherical rotor is translation combination of 3 degree of freedom in the calculation model. The $z$ axis indicated on Fig. 2 is the axle direction of a rocket, and the $x$ axis is the crossing at right angles direction.

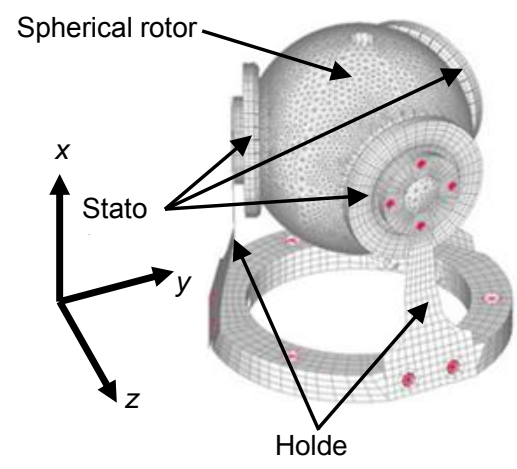

Fig. 2. Analysis model of SUSM.

The results of the natural vibration analysis to the calculation model are indicated in Table IV. The first natural 
frequencies of the SUSM in the axle direction of a rocket and the crossing at right angles direction is $598.71 \mathrm{~Hz}, 1422.64$ $\mathrm{Hz}$ respectively. The SUSM does not resonate with sine wave vibration of a rocket because the natural frequency of the SUSM is larger than the requested natural frequency (100 $\mathrm{Hz})$.

TABLE IV: RESULTS OF NATURAL VIBRATION ANALYSIS

\begin{tabular}{l|l|l}
\hline \hline Mode number & Natural frequency $(\mathrm{Hz})$ & Remarks \\
\hline 1 & 598.54 & $y$ primary \\
\hline 2 & 598.71 & $z$ primary \\
\hline 3 & 1133.21 & \\
\hline 4 & 1422.64 & $x$ primary \\
\hline 5 & 1569.09 & \\
\hline 6 & 1569.19 & \\
\hline 7 & 5084.35 & \\
\hline 8 & 5084.52 & \\
\hline 9 & 5490.16 & \\
\hline 10 & 6385.24 & \\
\hline \hline
\end{tabular}

\section{B. Resonance Search of SUSM}

Resonance search of the SUSM is performed using vibration exciter (VS-2000A-140T, IMV) and an acceleration sensor (VP-02S, IMV). The $z$ axis indicated on Fig. 3 is the axle direction of a rocket, and the $x$ axis is the crossing at right angles direction. For the part which vibrates most to be presumed as the holder, when the SUSM is vibrated in the $z$ axial direction, the measuring point of the amplitude of vibration of the $z$ axial direction is the holder part indicated on Fig. 4. On the other hand, for the part which vibrates most to be presumed as the top of the spherical rotor when the SUSM is vibrated in the $x$ axial direction, the measuring point of the amplitude of vibration of the $x$ axial direction is the upper part of the spherical rotor indicated on Fig. 4. Sine wave vibration of the constant acceleration 5.0 $\mathrm{m} / \mathrm{s}^{2}$ is given to each of the $z$ axial direction and the $\mathrm{x}$ axis direction. It was established as the frequency become double 2 minutes later. Resonance search from $5 \mathrm{~Hz}$ to $200 \mathrm{~Hz}$ and resonance search from $200 \mathrm{~Hz}$ to $2000 \mathrm{~Hz}$ are performed. The frequency of the vibration exciter when the transmissibility of the acceleration became biggest is the resonant frequency (the first natural frequency) of the SUSM.

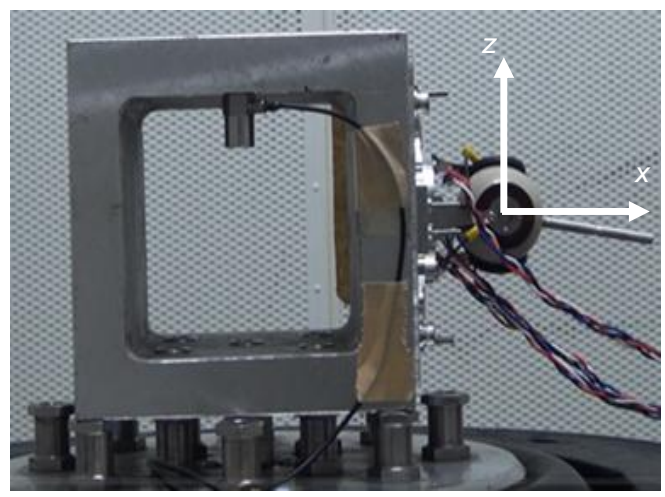

Fig. 3. SUSM on vibration machine.

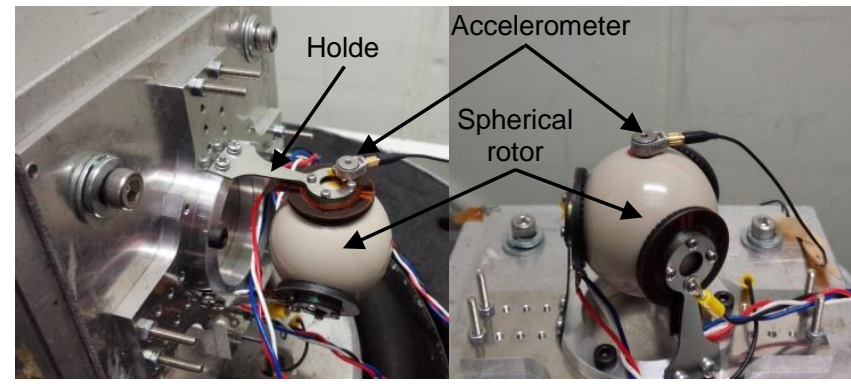

Fig. 4. Overview of resonance search of SUSM.

The relation between the frequency of the vibration exciter and the transmissibility of the acceleration obtained as a result of the resonance search of the $z$ axial direction and the $x$ axis direction is indicated on Fig. 5. And the transmissibility about the first natural frequency and the frequency in 2 directions is indicated in Table V. From Fig. 5, the first natural frequency of the axle direction and of the crossing at right angles direction in the SUSM is $625.0 \mathrm{~Hz}, 1530.0 \mathrm{~Hz}$ respectively. Therefore it was confirmed experimentally that the natural frequency of the SUSM is beyond $100 \mathrm{~Hz}$. The theoretical value, the experimental value and the relative error of the first natural frequency of the SUSM in 2 directions are indicated in Table VI.

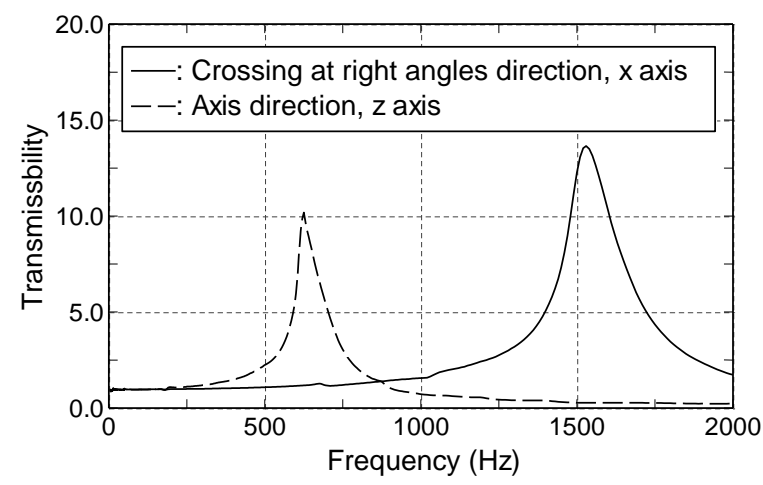

Fig. 5. Frequency - Transmissibility of acceleration.

TABLE V: PRIMARY NATURAL FREQUENCY AND TRANSMISSIBILITY

\begin{tabular}{l|l|l}
\hline \hline & $\begin{array}{l}\text { Primary natural } \\
\text { frequency }\end{array}$ & Transmissibility \\
\hline Axis direction & $625.0 \mathrm{~Hz}$ & 10.19 \\
\hline Crossing at right angles direction & $1530.0 \mathrm{~Hz}$ & 13.65 \\
\hline \hline
\end{tabular}

TABLE VI: PRIMARY NATURAL FREQUENCY OF ANALYSIS AND EXPERIMENT

\begin{tabular}{l|l|l|l}
\hline \hline & $\begin{array}{l}\text { Natural } \\
\text { vibration } \\
\text { analysis }\end{array}$ & $\begin{array}{l}\text { Resonance } \\
\text { search }\end{array}$ & Error \\
\hline Axis direction & $\begin{array}{l}598.71 \\
\mathrm{~Hz}\end{array}$ & $625.0 \mathrm{~Hz}$ & $4.23 \%$ \\
\hline Crossing at right angles direction & $\begin{array}{l}1422.64 \\
\mathrm{~Hz}\end{array}$ & $1530.0 \mathrm{~Hz}$ & $7.02 \%$ \\
\hline \hline
\end{tabular}




\section{Theoretical EVAluation By Design LOAD}

\section{A. Theory Equation for Resistance Against Vibration Evaluation}

Calculation of the intensity in the vibrational load at launch of a rocket is performed using the first natural frequency of the axle direction and the crossing at right angles direction in the SUSM obtained as a result of the resonance search. It is expected that the biggest load is added to the holder part of the SUSM by vibration. So calculation of the intensity to $a, b$, and $\mathrm{c}$ in the holder part indicated on Fig. 1 is performed. And calculation method of each design loads about the quasi-static acceleration, sine wave vibration, and random vibration are as following.

Design load $F_{q}$ in the quasi-static acceleration is expressed in (1):

$$
F_{q}=M \times G
$$

where, $M$ is the mass of the motor, and $G$ is the acceleration. From Table I, the acceleration added to the axle direction of a rocket is $6 \mathrm{G}$, and the acceleration added to the crossing at right angles direction is $5 \mathrm{G}$. And the mass of the motor is 160 g. When it is calculated using (1), design load $F_{q A}$ added to the axle direction in the quasi-static acceleration and design load $F_{q O}$ added to the crossing at right angles direction in the SUSM is $8.24 \mathrm{~N}, 6.87 \mathrm{~N}$ respectively.

The vibration condition to $100 \mathrm{~Hz}$ is prescribed by Table II about the loading condition of the sine wave vibration, and design load $F_{s}$ is calculated using the following (2):

$$
F_{S}=M \times Q \times G
$$

where, $Q$ is the resonance magnification defined by $1 /(2 \xi)$ (where, $\xi$ is the damping ratio). The numerical value of $Q$ is 1 because the first natural frequency of the SUSM is beyond $100 \mathrm{~Hz}$ in 2 directions. From Table II, the acceleration added to the axle direction in the SUSM is $2.5 \mathrm{G}$, and the acceleration added to the crossing at right angles direction in the SUSM is $2.0 \mathrm{G}$. When it is calculated using (2), design load $F_{S A}$ added to the axle direction of sine wave vibration, and design load $F_{S O}$ added to the crossing at right angles direction in the SUSM is $3.43 \mathrm{~N}, 2.75 \mathrm{~N}$ respectively.

The random vibration is expressed by the power spectrum density (PSD), and the probability density function of its amplitude is near normal distribution. The equation of Miles is used as a general way with a design of an artificial satellite. Design load $F_{r}$ is set by (3) as the equivalent stillness acceleration to which $3 \sigma$ of the normal distribution was applied:

$$
F_{r}=M \times 3 \sqrt{\frac{\pi}{2} \times Q \times f_{n} \times P S D_{n}},
$$

where, $f_{n}$ is the first natural frequency, and $P S D_{n}$ is the power spectrum density in the first natural frequency. As a result of the resonance search, the first natural frequency $f_{n A}$ of the axle direction in the SUSM is $625.0 \mathrm{~Hz}$, and first natural frequency $f_{n O}$ of the crossing at right angles direction in the SUSM is $1530.0 \mathrm{~Hz}$. It is necessary to consider about resonance magnification $Q$ because the first natural frequency is within $2000 \mathrm{~Hz}$ which is the vibration condition of the random vibration in each directions. The transmissibility of the acceleration in the resonant frequency indicated in Table $\mathrm{V}$ is used for resonance magnification $Q$. The vibrational acceleration is calculated by the second derivative of the amplitude about time. So the transmissibility of the acceleration in the resonant frequency is assumed as the transmissibility of the amplitude. From Table V, the resonance magnification $Q_{A}$ of the axle direction in the SUSM is 10.19, and the resonance magnification $Q_{O}$ of the crossing at right angles direction in the SUSM is 13.65. From Table III, when the frequency is $200 \mathrm{~Hz}-2000 \mathrm{~Hz}, P S D_{n}$ is $0.032 \mathrm{G}^{2} / \mathrm{Hz}$. And when it is calculated using (3), the design load $F_{r A}$ added to the axle direction of the random vibration and the design load $F_{r O}$ added to the crossing at right angles direction of the random vibration is $84.3 \mathrm{~N}, 153.0 \mathrm{~N}$ respectively.

\section{B. Result of Theoretical Calculation}

When launching the SUSM by a rocket actually, a design load by vibration is additional value of all design loads calculated using (1)-(3). The whole design load $F_{\text {all }}$ of the axle direction of a rocket and the whole design load $F_{\text {allo }}$ of the crossing at right angles direction is $95.9 \mathrm{~N}, 162.0 \mathrm{~N}$ respectively. The numerical value into which $F_{\text {allA }}$ and $F_{\text {allo }}$ are divided by a cross-sectional area of a, b, and $\mathrm{c}$ in the holder part are design stress $\sigma_{s}$ in each locations. When margin of safety $M S$ calculated using design stress $\sigma_{s}$ and permissible stress $\sigma_{a}$ by the material of the holder part and the screw part satisfies the following (4), the holder part and the screw part do not transform by vibration:

$$
M S=\sigma_{a} / \sigma_{s}-1 \geq 0
$$

After $M S$ was calculated about 3 points of $\mathrm{a}, \mathrm{b}$, and $\mathrm{c}$ in the holder part, all $M S$ satisfied (4). Therefore the holder part and the screw part do not transform by vibration. On the other hand, for the shock load to be added to the SUSM momentarily about calculation of the intensity of shock resistance, it is assumed that the load beyond the maximum acceleration is added to the SUSM. Therefore shock resistance is evaluated experimentally because it is difficult to carry out calculation of the intensity using a theory equation.

\section{EXPERIMENTAL EVALUATION OF RESISTANCE AgAINST VIBRATION AND SHOCK RESISTANCE}

\section{A. Outline of Experiment}

To investigate resistance against vibration and shock resistance, the SUSM is installed in the vibration exciter and is carried out experiment of resistance against vibration and shock resistance. The vibration direction is same as resonance search. The stimules experiments of the $z$ axial direction and the $x$ axis direction are carried out about quasi-static acceleration and sine wave vibration, and random vibration. And it is confirmed about drive of the SUSM after stimules. The SUSM is disassembled after each experiment 
and damage of all parts is checked. Each experiment of resistance against vibration and shock resistance are described to below.

\section{B. Experiment of Resistance Against Vibration}

In the experiment of resistance against vibration by the quasi-static acceleration, the sine wave vibration is $8 \mathrm{~Hz}$, and the acceleration is given $5.0 \mathrm{G}$ to the $z$ axial direction and 6.0 $\mathrm{G}$ to the $x$ axis direction for 2 minutes.

In the experiment of resistance against vibration by the sine wave vibration, the frequency is changed from $5 \mathrm{~Hz}$ to $100 \mathrm{~Hz}$. The sine wave vibration is given $2.5 \mathrm{G}$ to the $z$ axial direction and $2.0 \mathrm{G}$ to the $x$ axis direction.

In the experiment of resistance against vibration by the random vibration, the acceleration density is $+3 \mathrm{~dB} /$ oct to $20-200 \mathrm{~Hz}$, and is $0.032 \mathrm{G} 2 / \mathrm{Hz}$ to $200-2000 \mathrm{~Hz}$ to the $z$ axial direction and the $x$ axis direction for 2 minutes. The effective value is $7.8 \mathrm{G}$.

\section{Experiment of Shock Resistance}

In the experiment of shock resistance by shock acceleration of $20 \mathrm{G}$, the maximum acceleration is $20 \mathrm{G}$, and the sine wave vibration of the half wave which half wave time is $5 \mathrm{~ms}$ is given the $z$ axial direction and the $x$ axis direction.

In the experiment of shock resistance by shock acceleration of $30 \mathrm{G}$, the maximum acceleration is $30 \mathrm{G}$, and the sine wave vibration of the half wave which half wave time is $11 \mathrm{~ms}$ is given the $z$ axial direction and the $x$ axis direction.

In the experiment of shock resistance by shock acceleration of $40 \mathrm{G}$, the maximum acceleration is $40 \mathrm{G}$, and the sine wave vibration of the half wave which half wave time is $11 \mathrm{~ms}$ is given the $z$ axial direction and the $x$ axis direction.

\section{Experimental Result}

After each stimules experiments, it was checked about drive of the SUSM. A crack and transformation were not seen by the holder part and the screw part, but it was confirmed to have several small cracks on the rubbing surface of the spherical rotor and the stator.

During the experiment of the random vibration, it was observed that a spherical rotor was moving slowly. The spherical rotor is maintained by frictional force and pressing force of three stators in a fixed position. It is considered as the cause that holding torque could not be maintained because frictional force fell at the rubbing surface by the small amplitude and the high frequency in the random vibration.

There was no leaving from the holder of the spherical rotor by a shock in the experiment of shock resistance by shock acceleration of $20 \mathrm{G}$ and $30 \mathrm{G}$ and the drive of the SUSM was confirmed. On the other hand, there was leaving from the holder of the spherical rotor by a shock in the experiment of shock resistance by shock acceleration of $40 \mathrm{G}$ in the $x$ axis direction. The stopper was made as the countermeasure to prevent leaving of the spherical rotor. And when the experiment of shock resistance was carried out again using the SUSM with the stopper, leaving of the spherical rotor was not observed in shock acceleration of $40 \mathrm{G}$, and the drive of the SUSM was confirmed.

The rotational speed of the SUSM before and after the endurance experiment is indicated in Table VII and the impedance of the piezoelectric element before and after the endurance experiment is indicated in Fig. 6. It was confirmed that there are no remarkable changes in the rotational speed from Table VII. It was confirmed that the characteristic of the impedance is also obtained from Fig. 6 after the experiment. Therefore it was confirmed that there are no declines in the drive performance of the SUSM by vibrational acceleration at launch of a rocket and shock acceleration of $40 \mathrm{G}$ of a rocket.

TABLE VII: ROTATIONAL SPEED BEFORE AND AFTER EXPERIMENT

\begin{tabular}{l|l|l}
\hline \hline & Before & After \\
\hline Rotational speed (rpm) & 44.7 & 49.7 \\
\hline \hline
\end{tabular}

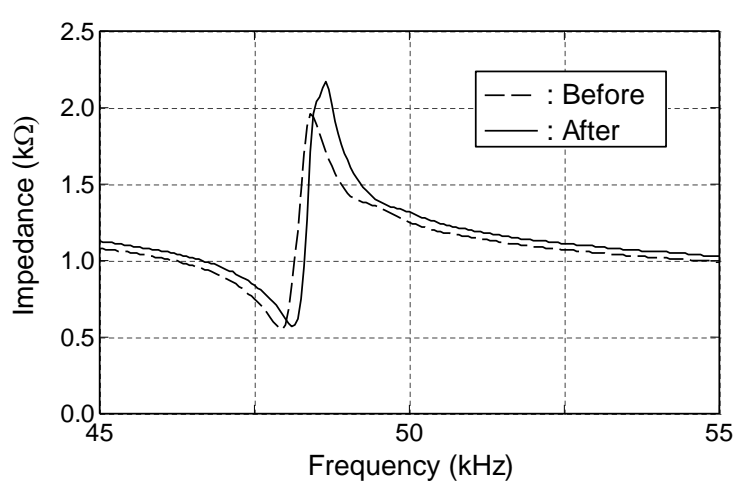

Fig. 6. Impedance characteristics before and after experiment.

\section{CONCLUSIONS}

It was confirmed that the first natural frequency of the SUSM is beyond $100 \mathrm{~Hz}$ of the required specification by performing the characteristic vibration analysis and resonance search. The SUSM was evaluated theoretically and experimentally about resistance against vibration, and it was confirmed that there are no problems. And when the SUSM received shock acceleration of $40 \mathrm{G}$, it was confirmed experimentally that the SUSM is able to endure shock acceleration.

However, there is a big shock load which occurs by an explosion of gunpowder in the load added to an artificial satellite. When the big shock load was given, it is necessary to change the holder part and the screw part to the strong high material so that the SUSM may endure its load.

\section{ACKNOWLEDGMENT}

This work was supported by JSPS KAKENHI Grant Number JP16K06060.

\section{REFERENCES}

[1] S. Toyama, S. Sugitani, G. Zhang, Y. Miyatani, and K. Nakamura, "Multi degree of freedom spherical ultrasonic motor," in Proc. 1995 IEEE International Conference on Robotics and Automation, pp. 2935-2940, 1995.

[2] E. Purwanto and S. Toyama, "Control method of a spherical ultrasonic motor," in Proc. 2003 IEEE/ASME International Conference on Advanced Intelligent Mechatronics, pp. 1321-1326, 2003.

[3] N. Takesue, T. Ohara, R. Ishibashi, S. Toyama, M. Hoshina, Y. Hirai, N. Fukaya, J. Arata, and H. Fujimoto, "Position control methods of spherical ultrasonic motor," in Proc. 2010 IEEE/RSJ International Conference on Intelligent Robots and Systems, pp. 3061-3066, 2010.

[4] Z. Guo, Y. Bai, L. Yi, J. Guo, and J. Wang, "A novel method on real-time measurement of 2-DOF motions of spherical ultrasonic motor," Applied Mechanics and Materials, vol. 416-417, pp. 1080-1085, 2013. 
[5] T. Oohashi and S. Toyama, "Development of spherical ultrasonic motor for space," Applied Mechanics and Materials, vol. 555, pp. 26-31, June 2014.

[6] U. Nishizawa, T. Oohashi, and S. Toyama, "Evaluation of spherical ultrasonic motor for space in high temperature condition," Journal of Vibroengineering, vol. 18, issue 2, pp. 916-926, Mar. 2016.

[7] U. Nishizawa, T. Oohashi, and S. Toyama, "Evaluation of spherical ultrasonic motor for space in low temperature condition," Journal of Vibroengineering, vol. 19, no. 7, pp. 5170-5181, Nov. 2017.

[8] T. Sashida and T. Kenjo, An Introduction to Ultrasonic Motors, Oxford: Oxford University Press, 1993

[9] A. Ferreira and P. Minotti, "High-performance load-adaptive speed control for ultrasonic motors," Control Engineering Practice, vol. 6, no 1, pp. 1-13, 1998.

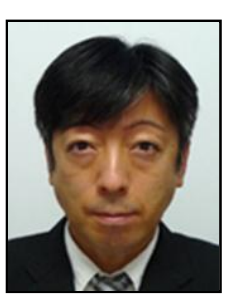

Uichi Nishizawa received Ph.D. degree in engineering from Tokyo University of Agriculture and Technology, Tokyo, Japan, in 2008. Now he works at Tokyo University of Agriculture and Technology. His current research interests include robotics, aerospace engineering, and welfare technology.

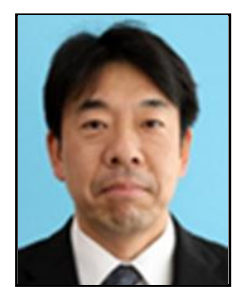

Taro Oohashi received master degree in engineering from Akita University, Akita, Japan, in 1993. Now he works at Kisarazu National College of Technology.

His current research interests include control and tribology.

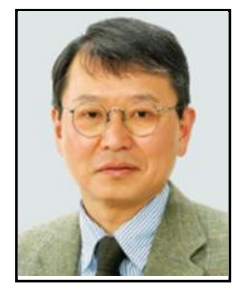

Shigeki Toyama received Ph.D. degree in Engineering from University of Tokyo, Tokyo, Japan, in 1981. Now he works at Tokyo University of Agriculture and Technology. His current research interests include robotics, actuator, and welfare technology. 